\title{
Shock Motion and Flow Instabilities in Supersonic Nozzle Flow Separation
}

\author{
Andrew Johnson ${ }^{1}$ and Dimitri Papamoschou ${ }^{2}$ \\ University of California, Irvine, Irvine, CA, 92697-3975
}

\begin{abstract}
This study evaluates the role of shock wave motion on the instability of the plume exiting an over-expanded, convergent-divergent nozzle. An array of wall pressure transducers was used to track the position of the shock in time, and a Pitot probe was used to obtain simultaneous measurement of the total pressure fluctuations at various points in the jet that emerges from the separation shock. Analysis of the shock motion revealed that the shock wave becomes more unstable as it becomes stronger, as evidenced by an increase in the range of motion and in the frequency of large-scale oscillations. For strong shocks, there is a substantial correlation between shock motion and total pressure fluctuation in the plume. Such correlation is absent for relatively weak shocks. The study indicates that shock motion affects the plume instability if the separation shock is very strong, and that other mechanisms govern the plume instability when the shock is relatively weak.
\end{abstract}

\section{Nomenclature}
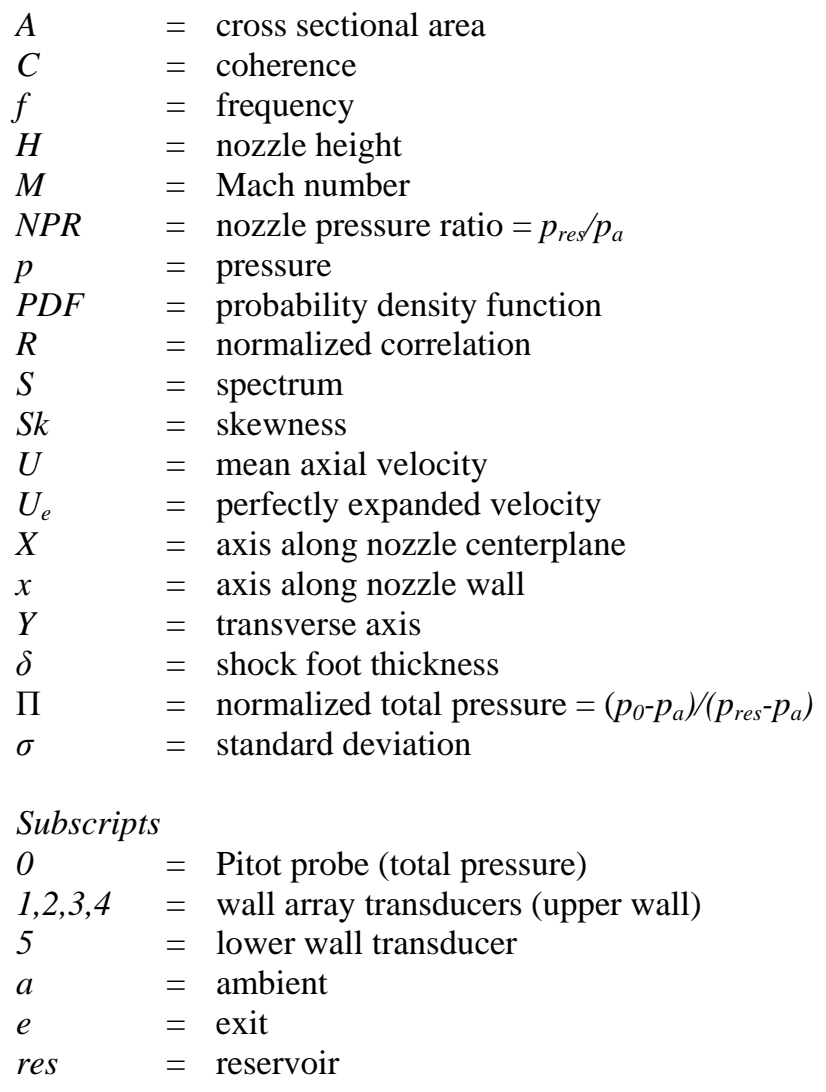

${ }^{1}$ Graduate Student Researcher, Department of Mechanical and Aerospace Engineering, 4200 Engineering Gateway, Irvine, CA, 92697-3975, AIAA Member.

${ }^{2}$ Professor, Department of Mechanical and Aerospace Engineering, 4200 Engineering Gateway, Irvine, CA, 926973975, AIAA Associate Fellow. 


$\begin{array}{ll}r m s & =\text { root mean square } \\ s & =\text { shock } \\ t & =\text { throat }\end{array}$

\section{Introduction}

SHOCK formation inside a convergent-divergent nozzle introduces instabilities in the internal and external flows. The external-flow instability can be used as a means of intensifying mixing in the nozzle plume itself (Fig. 1), or as a means of destabilizing an adjacent stream (Fig. 2). Both applications have been investigated at U.C. Irvine, where the phenomenon was discovered, and in other institutions under the general concept of Mixing Enhancement using Axial Flow (MEAF). ${ }^{1,2,3}$ While the benefits to mixing are quite obvious, the mechanisms that generate this instability are obscured by the complexity of the internal nozzle flowfield, shown schematically in Fig. 3. This study builds on previous experimental and numerical studies of this phenomenon. Its specific purpose is to investigate the relationship between the shock motion and the instability of the plume that emerges from the separation.

We begin with a brief description of the principal separation phenomena in a symmetric nozzle, shown schematically in Fig. 4. $\quad$ Near the wall, the separation shock consists of incident and reflected oblique waves that merge into a Mach stem at the triple point. This is the so-called lambda foot of the shock. The adverse pressure gradient through the shock forces the incoming boundary layer to separate, forming separation regions downstream. The oblique shock structures are of the "weak" type resulting in low supersonic flow downstream while the flow immediately past the Mach stem is subsonic. Wavy slipstreams emerge from the triple points that form a convergent-divergent fluidic channel, which acts to reaccelerate the subsonic flow downstream of the normal portion of the shock back to supersonic, as illustrated in the schematic of Fig. 4. The trailing shocks reflect off of the separation shear layers emerging as expansion fans that are then transmitted across the test section to the opposite shear layers where they are reflected again as compression waves. These reflections continue downstream, resulting in a series of alternating regions of expansion and compression through the separation jet.

At high nozzle pressure ratios $\left(\mathrm{NPR}=p_{\text {res }} / p_{a}\right)$ and exit-to-throat area ratios $\left(A_{e} / A_{t}\right)$ the separation occurs asymmetrically with one lambda foot being larger than the other. ${ }^{4}$ A collaborative investigation by Xiao et al. ${ }^{5}$ successfully predicted this asymmetry numerically by solving the Reynolds Averaged Navier Stokes equations with a two equation $k-\omega$ turbulence model. This study also showed a rapid expansion downstream of the Mach stem followed by compression, further supporting the model of Fig. 4. When separation occurs asymmetrically, the orientation of the asymmetry is steady during a given experiment. The orientation can change from one experiment to the next suggesting that it is sensitive to the startup conditions.

The source of unsteadiness in shock-boundary layer interaction has been studied in a variety of flowfields and yet remains a widely debated subject. Even though some experiments and analyses have shown that the shock motion can be influenced by turbulent fluctuations in the attached boundary layer upstream, ${ }^{6,7,8}$ it would seem that in general, the primary excitation comes from fluctuation in the downstream separated flow. This is reflected in a comprehensive review of several experiments by Dussauge et al. ${ }^{9}$ In an investigation motivated by the occurrence of side loads in rocket nozzles, Bourgoing and Reijasse ${ }^{10}$ studied unsteady shock behavior in a two-throated planar nozzle undergoing both symmetric and asymmetric separation. The presence of the second throat resulted in the reattachment of the recirculation regions downstream of the shock. Wall pressure spectra measured near the recirculation regions showed two spectral bumps. Dussauge ${ }^{9}$ suggests that the lower and higher frequency bumps may be associated with the finite length of the larger and smaller recirculation zones respectively. This notion is supported by the relatively broadband wall pressure spectra obtained in the experiments at UC Irvine, in which the separation zones downstream of the main shock did not reattach. ${ }^{4,11,12}$

Because the motivation behind this investigation is mixing enhancement, our primary focus is not the source of the shock unsteadiness but rather the impact of the shock motion on the flow downstream; specifically, its contribution to the instability of the separation shear layers. Papamoschou and Zill ${ }^{4}$ observed increased instability of the large separation shear layer during asymmetric separation, with large eddies sometimes occupying half of the test section. Mean flow surveys of the nozzle plume ${ }^{12}$ showed that the growth rate of the jet plume nearly doubled for cases where the separation occurred asymmetrically. The growth-rate increase was accompanied by a three-fold increase in the peak rms level of the total pressure fluctuation at the exit of the nozzle Numerical predictions using a shear stress transport (SST) turbulence model showed close agreement with these experimental results with increased levels of turbulent kinetic energy near the nozzle exit and improved mixing in the plume for cases where asymmetric separation occurred. ${ }^{13}$ 
Experiments by Papamoschou and Johnson, ${ }^{11}$ using time-resolved pressure measurements, showed that the total pressure fluctuation in the large separation shear layer correlates well with wall pressure fluctuations measured at the mean shock location, indicating a possible connection between shock unsteadiness and shear-layer instability. However, the conditions of those experiments were limited and reliance on a single probe to determine shock motion is unsatisfactory. Further, an additional source of instability may be the alternating succession of expansion and compression waves downstream of the main shock. While numerous studies have focused on the receptivity of a shear layer to a field of alternating compressions and expansions, these studies have generally considered cases of higher Mach numbers than are relevant here. ${ }^{14,15}$ Thus, no fore drawn conclusions can be made about the impact of the downstream wave pattern. It should be noted that the small separation shear layer is subject to the same physics as the large separation shear layer. This suggests that the asymmetric configuration provides the space for instability waves in the larger separation shear layer to grow while growth in the small separation shear layer is suppressed.

The experiments conducted in the current investigation were designed to evaluate the unsteady shock motion in order to assess its role in the instability in the large separation shear layer. The overall objective was to obtain an improved understanding of the underlying physics in supersonic nozzle flow separation so that it can be utilized more effectively for practical applications in high speed mixing.

\section{Experiment}

\section{A. Nozzle Facility}

The facility utilized in this investigation was designed at UC Irvine to allow versatility in the study of nozzle flows. The facility, shown in Fig. 5, incorporates two flexible plates forming the upper and lower walls of the nozzle that can be shaped using two sets of actuators located at the end of each plate. Adjusting the transverse force and moment applied to each wall allows variation in the exit-to-throat area ratio $\left(A_{e} / A_{t}\right)$, nozzle contour, and maximum wall angle. The sidewalls of the nozzle test section consist of large optical windows to provide visualization of the entire internal flowfield. The nominal test section dimensions are $17.8 \mathrm{~mm}$ in height, $63.5 \mathrm{~mm}$ in width, and $117 \mathrm{~mm}$ in length from throat to exit. The apparatus is supplied by a pressure-regulated air supply capable of obtaining nozzle pressure ratios $\left(\mathrm{NPR}=p_{\text {res }} / p_{a}\right)$ up to 3.5 allowing for comprehensive study of various nozzle flows from subsonic, to shock containing, to shock-free flow.

\section{B. Diagnostics}

This investigation utilized time-resolved pressure measurements of the nozzle wall pressure and of the total pressure in the flow downstream of the shock. All the measurements used piezoelectric pressure transducers (Endevco Model 8507C-15) with 2-mm diameter sensors and frequency response of $30 \mathrm{kHz}$. Fig. 6 provides the layout of the transducers.

Four transducers were mounted flush along the upper wall of the nozzle and were equally spaced to span a total of distance of $12.7 \mathrm{~mm}$ from the center of the first probe to the center of the final probe. This distance corresponded to the estimated range of shock motion for the configurations of interest based on schlieren photography. The resulting array provides the instantaneous wall pressure distribution caused by the shock foot which is then used to track the longitudinal position of the shock foot along the nozzle wall. The details of this tracking procedure are provided in the following section. A fifth probe was mounted on the lower wall opposite the first probe of the upper-wall array. In addition to providing valuable quantitative information about the fluctuating flowfield, the fifth wall probe measurement was used in conjunction with the wall array measurement to verify the orientation of the shock asymmetry for a given run by exploiting the fact that the larger lambda foot extends further upstream than the smaller lambda foot.

A dynamic Pitot probe was used to measure the impact pressure, and its fluctuations, downstream of the shock. Given that this flow field is mildly supersonic at most, the impact pressure is practically identical to the total pressure $p_{0}$. In the absence of time-resolved velocity measurement (which are very difficult for this high-speed flow field), we use the fluctuations in total pressure to characterize the unsteadiness of the flow. The dynamic Pitot probe, depicted in Fig. 7, consists of a piezoelectric transducer oriented against the flow with an S-shaped protective inlet that prevents collision of particulates in the air with the transducer face. The length of the inlet is $8 \mathrm{~mm}$ and its inner diameter is $1.7 \mathrm{~mm}$. The inlet introduces distortions in the frequency response of the probe that need to be accounted for when calculating statistics such as the autospectra and variance of $p_{0}$ '. The distortion was determined by extensive testing of the transducer, with and without the protective inlet, in stagnation-point flows with various amplitudes of pressure fluctuations. For all amplitudes, the distortion spectrum peaked at $10.7 \mathrm{kHz}$. Correction procedures, detailed in Ref. 11, are implemented for removing the distortion from the autospectrum of the pressure fluctuations and from the resulting computation of the variance. 
All six transducers were sampled simultaneously at a sampling rate of $200 \mathrm{kS} / \mathrm{s}$ per channel for the duration of 1 second. A fourth-order low pass filter with $80 \mathrm{kHz}$ corner frequency was installed in the amplifier to prevent effects of aliasing. Spectra were computed using a 4096-point Fast Fourier Transform resulting in a frequency resolution of $48.8 \mathrm{~Hz}$. Cross-correlations are presented in a normalized fashion.

\section{Flow Conditions}

The experiment involved four combinations of nozzle area ratio and pressure ratio that resulted in a progressively stronger shock situated at the midpoint of the upper-wall transducer array (Fig.6). This corresponds to a mean shock position of approximately three throat heights upstream of the nozzle exit $\left(X / H_{t}=-3.0\right)$. The combinations of NPR and $A_{e} / A_{t}$, and resulting shock strength, are listed in Table 1 . The shock strength is given by the normal shock relation

$$
\frac{\Delta p}{p}=\frac{2 \gamma}{\gamma+1}\left(M_{s}^{2}-1\right)
$$

with the shock Mach number $M_{s}$ based on the mean area ratio where the shock sits. The Reynolds number based on throat height and perfectly expanded exit conditions ranged from $3.53 \times 10^{5}$ (Case 1) to $5.23 \times 10^{5}$ (Case 4).

In all experiments, the upper wall is associated with the large separation region and the lower wall with the small separation region.

Table 1. Test Configurations

\begin{tabular}{|c|c|c|c|}
\hline Case & $\begin{array}{c}\text { Nozzle Area Ratio } \\
\boldsymbol{A}_{\boldsymbol{e}} / \boldsymbol{A}_{\boldsymbol{t}}\end{array}$ & $\begin{array}{c}\text { Nozzle Pressure Ratio } \\
\mathbf{N P R}=\boldsymbol{p}_{\text {res }} / \boldsymbol{p}_{\boldsymbol{a}}\end{array}$ & $\begin{array}{c}\text { Shock Strength } \\
\boldsymbol{\Delta} / \boldsymbol{p}\end{array}$ \\
\hline 1 & 1.4 & 1.45 & 1.75 \\
\hline 2 & 1.5 & 1.55 & 1.90 \\
\hline 3 & 1.6 & 1.70 & 2.01 \\
\hline 4 & 1.7 & 1.90 & 2.17 \\
\hline
\end{tabular}

\section{Tracking of Shock Position}

In this section we describe tracking of the shock motion using the instantaneous wall pressure measurements of the upper-wall transducer array of Fig. 6. The wall pressure "footprint" of the separation shock has a finite region because of its lambda-foot structure. Consequently, the wall-pressure distribution across the shock is smooth. Considering the narrow vicinity around the shock, we can approximate this pressure distribution using a hyperbolic tangent function of the form,

$$
\mathrm{P}\left(x, x_{s}\right)=\frac{p_{s 2}+p_{s 1}}{2}+\frac{p_{s 2}-p_{s 1}}{2} \tanh \left[\frac{2}{\delta}\left(x-x_{s}\right)\right]
$$

The parameters of this distribution are the pressures $p_{s 1}$ and $p_{s 2}$ before and after the jump, respectively, the thickness of the distribution $\delta$, and the shock position $x_{s}$. The distribution is illustrated in Fig. 8a, and the definition of thickness in Fig. 8b. The factor of 2 in the argument of the tanh is consistent with the definition of thickness given below.

The pressure transducers provide $p\left(x_{i}\right)=p_{i}$ for a given time at their four discrete locations, $x_{1} \ldots x_{4}$. We use the experimental measurements of $p_{i}$ to fit the distribution of Eq. 1 using a nonlinear least-squares scheme. The amplitudes $p_{s 1}$ and $p_{s 2}$ normally correspond to the instantaneous measurements of the minimum and maximum values of the array. However, for the rare occasion when the shock position $x_{s}$ is outside the spatial range of the array, the amplitudes are based on the mean (time-averaged) pressures at the first and last probes. Because the shock primarily oscillates within the spatial range of the array, the mean pressures of the first and last probe approximately reflect the mean pressures in the attached flow upstream and separated flow downstream of the shock, respectively. This provides a logical method for extrapolating the model pressure distribution beyond the span of the array without having the actual measurements for the pressure before and after the jump. The mean pressure gradient in the attached boundary layer upstream of the shock is negative, and therefore it is reasonable to assume that the minimum pressure from the transducer array will not exceed the mean pressure of the first 
transducer unless the shock wave has moved past upstream boundary of the array, $x_{1}$. The resulting fitting scheme for $p_{s 1}$ is given in Eq. 2. A similar argument can be used to justify the fitting scheme for $p_{s 2}$ shown in Eq. 3 . The mean pressure of the fourth transducer should not exceed the maximum instantaneous pressure measured by the array unless the shock wave has moved past the downstream boundary of the array, $x_{4}$, or if there is a strong negative fluctuation in pressure measured by the fourth transducer due to turbulence in the separated region. For either situation, setting $p_{\mathrm{s} 2}$ equal to the mean pressure of the fourth transducer will provide a more accurate measurement of the shock position.

$$
\begin{aligned}
& p_{s 1}=\min \left\{\begin{array}{c}
p_{i, \min } \\
\bar{p}_{1}
\end{array}\right\} \\
& p_{s 2}=\max \left\{\begin{array}{c}
p_{i, \max } \\
\bar{p}_{4}
\end{array}\right\}
\end{aligned}
$$

The thickness $\delta$ is based on the maximum gradient between any two consecutive points in the measured distribution as follows:

$$
\delta=\left(p_{s 2}-p_{s 1}\right) /\left.\left(\frac{p_{i+1}-p_{i}}{x_{i+1}-x_{i}}\right)\right|_{\max }
$$

The minimization process requires that we first guess the position of the shock, $x_{s}$, and then correct this value iteratively until the error between the theoretical and actual pressure distributions is minimized. To obtain the appropriate correction we define the error vector.

$$
e_{i}=p_{i}-\mathrm{P}\left(x_{i}, x_{s}\right)
$$

Next we obtain a linearized estimate for the correction, $d x_{s}$ that minimizes the error vector by computing

$$
d x_{s}=J_{i}^{-1} e_{i}
$$

where $J$ is the Jacobian

$$
J_{i}=\frac{\partial}{\partial x_{s}} \mathrm{P}\left(x_{i}, x_{s}\right)
$$

This correction is then applied to the original guess, via Eq. 8, in order to obtain a new guess for the next iteration. The coefficient $\lambda$ is an under-relaxation factor that can be applied to provide stability to the computation if necessary.

$$
x_{s}=x_{s}+\lambda d x_{s}
$$

Once $x_{s}$ is updated, the process is repeated through successive iterations until the error reaches a minimum. Since the minimum error may differ from one time instance to the next and its value is not known a priori, we define the following residual to determine whether the solution has converged.

$$
r \equiv\left|E_{n+1}-E_{n}\right|
$$

where the error function $E$ is defined by

$$
E \equiv \sum_{i=1}^{4} e_{i}^{2}
$$

Convergence was based on a set limit of $r \leq 10^{-10}$.

Figs. 9a and 9b show a typical shock position trace and probability density function obtained using this method for a nozzle area ratio of $A_{e} / A_{t}=1.4$ and a nozzle pressure ratio of NPR=1.45 (Case 1). A necessary validation of the tracking method is that resulting position signal is negatively correlated to the pressure signal from one of the probes in the wall array. This results from the fact that as the shock moves upstream across a given transducer (negative $x$ direction), the probe experiences a pressure increase and vice-versa. Fig.10 shows the cross-correlation between the shock position fluctuation, as measures using the method describe above, and the wall pressure fluctuation measured 
by transducer 2 in the wall probe array. There is a strong correlation between these two signals, providing reassurance that the tracking method works properly.

\section{Results}

\section{A. Statistics of Shock Motion}

The shock position trace and probability density function for Case 1 are shown in Figs. 9a and 9b, respectively. For this relatively weak shock, separation is weakly asymmetric. The shock position signal is characterized by small-scale oscillations about the preferred, or equilibrium shock position, with occasional large-scale perturbations primarily in the upstream direction. This behavior is reflected in the asymmetry of the probability density function, where the peak value occurs at a normalized position that is greater than zero. This means that the equilibrium shock location is slightly further downstream than the mean shock location. The noticeable skewness in the plot of Fig. 9a $\left(\mathrm{Sk} / \sigma^{3} \approx-0.8\right)$ is the result of the occasional large scale fluctuation toward the upstream direction. Overall, this behavior would seem to support the argument that the source of unsteadiness comes from downstream of the shock.

As the nozzle area ratio and nozzle pressure ratio are increased, the shock becomes stronger. The resulting effect on the shock motion can be seen in the position trace of the Fig. 11 for Case 3. There is an obvious increase in both the range of the shock wave motion as well as the frequency of large scale motions. The result is an increase in the root means square of the fluctuation and a reduction in the magnitude of the skewness of the signal $\left(\mathrm{Sk} / \sigma^{3} \approx-0.3\right)$ Thus, the shock becomes more unstable with increasing strength. This trend has been noticed in various other shockcontaining flowfields. ${ }^{8}$ We quantify these trends by plotting the rms and peak-to-peak amplitudes of the shock location versus shock strength in Figs. 12 and 13, respectively. For the stronger shocks, the peak-to-peak amplitude of shock motion is of the same order as the nozzle throat height.

Spectra of the shock position fluctuation and of the wall pressure fluctuation near the mean shock location for Case 3 are plotted in Figs. 14a and b. Both spectra are broadband and their shapes are similar. It is important to note that the spectra are smooth with no peaks or bumps such as the ones noticed in the experiments by Bourgoing and Reijasse. ${ }^{9}$ This implies that the unsteadiness is not governed by any resonant interactions, but rather by random fluctuations.

\section{B. Correlations between Shock Motion and Plume Total Pressure}

In this section we study correlations between the shock motion and the fluctuating total pressure as measured by the dynamic Pitot probe at various points in the separated flow. The coordinate system and positions of the Pitot probe are shown in Fig. 15. We first consider the nature of the fluctuations downstream of the shock. Fig. 16 shows the spectrum of total pressure fluctuations for Case 3 at the exit of the nozzle near the large separation shear layer $\left(X / H_{t}=0.0, Y / H_{t}=0.32\right)$. The shape of the spectrum is not unlike that of the shock position spectrum shown in Fig. 14a, where the highest spectral intensity occurs at low frequency. The primary difference in the shape of the spectra is the slight increase in intensity in the mid to high frequency range of the total pressure spectrum, which may be associated with the presence of broadband turbulent fluctuations in the shear layer.

For the following correlations, the Pitot probe was at a fixed position one exit height upstream of the nozzle exit, measuring in the large separation shear layer downstream of the shock $\left(X / H_{t}=-1, Y / H_{t}=0.32\right)$. First consider Case 1 , where the shock motion is relatively weak. Fig. 17a shows the correlation of the shock motion with the total pressure fluctuation. It is practically zero, indicating no significant interaction. In Case 3, where the shock motion is strong with frequent large amplitude motions, the correlation becomes substantial, as shown in Fig. 17b. The frequency content of this cross-correlation can be found by plotting the coherence between the shock motion and the total pressure fluctuations, presented in Fig. 18. It is obvious from the plot that the correlation is a low frequency phenomenon (less than a non-dimension frequency of 0.2). From the shock motion spectrum of Fig. 14a we see that this frequency domain corresponds to the large amplitude motions of the shock, which explains the absence of correlation in Case 1.

Given that the large-scale motions of the shock become more frequent as the shock becomes more unstable (i.e. at high values of nozzle pressure ratio nozzle area ratio), it should then be expected that the correlation between the shock position fluctuation and total pressure fluctuations in the large separation shear layer becomes stronger from Case 1 to Case 4. To verify this, the Pitot probe was traversed through the internal and external flows according to the grid of Fig. 15. We define the correlation "peak" as the maximum value of the correlation, if it is positive, or its minimum value, if it is negative. Figures 19 a-d show contour plots of the correlation peaks for Cases 1 to 4, respectively. The contour plots include the outline of the nozzle (solid lines) and the approximate position of the shock (dashed lines). 
As the shock strength increases the correlation between shock motion and total pressure fluctuation in the separation jet becomes stronger and extends over a larger spatial range. For Case 4, the peak correlation is as high as 0.4 at two throat heights downstream of the nozzle exit. An unexpected result however, is the negative correlation present when the Pitot probe is located near the centerline of the nozzle in the separation jet. This is especially noticeable in Fig. 19c for Case 3. One possible explanation for this correlation is that when the shock moves downstream (positive $x$-direction) it becomes stronger (higher total pressure loss), resulting in a negative perturbation of the total pressure downstream; the opposite being true when the shock is perturbed upstream. In case 4, the negative correlation becomes weaker and confined near the lower wall of the nozzle, as seen in Fig. 19d. This is possibly the result of the instability of the large-separation shear layer becoming so strong that it dominates most of the nozzle flow.

Analogous contours of the normalized mean total pressure are plotted over the same domain for Cases 3 and 4 in Fig. 20a and 20b, respectively. Comparing with the correlation plots of Figs. 19c and 19d it is apparent that the correlation of shock motion and total pressure fluctuation in the shear layer peaks near the dividing streamline. This suggests that the large-scale shock motion influences strongly the spatial evolution of the shear layer. Since the shock defines the origin of the shear layer, one could think of the shock motion as a means of forcing (analogous perhaps to moving the splitter plate tip in a shear layer experiment) that can increase the instability and growth rate of the shear layer. The response of the shear layer to this excitation will depend on the nature of the excitation. Our experiment suggests low receptivity if the shock motion is not very strong, and significant receptivity if the shock motion is large. It appears, therefore, that shock motion contributes to the plume instability when the shock oscillation is substantial.

\section{Conclusion}

We have conducted an investigation in order to assess the impact of unsteady shock wave motion in supersonic nozzle flow separation on observed instability in the jet plume downstream of the separation. Progressively stronger shocks were created by increasing the nozzle area ratio and pressure ratio. The separation was always asymmetric, with the shock having a large lambda foot on one side (large separation shear layer) and a small lambda foot on the other side (small separation shear layer). The instantaneous position of the large lambda foot was tracked using an array of piezoresistive pressure transducers mounted flush on the wall of the nozzle. Simultaneous measurements of total pressure fluctuation in the downstream flow were obtained using a dynamic Pitot probe. The key findings are as follows.

The amplitude of shock motion increases with shock strength. The motion is broad-band with significant lowfrequency content. The total pressure fluctuations in the separated flow are broad-band as well. A strong correlation between the shock motion and total pressure fluctuation in the large separation shear layer is observed for shock strength $4 p / p \geq 2.0$ (Cases 3 and 4). The coherence of the signals indicates that the correlation is associated with low-frequency (large-scale) motions of the shock. The correlation peaks near the dividing streamline of the large shear layer suggesting that the shock motion acts as a forcing function driving the instability. A weaker, negative correlation is observed towards the side of the small shear layer.

On the other hand, no significant correlations between shock motion and plume total pressure were measured for $\Delta p / p \leq 1.9$ (Cases 1 and 2). Past experiments have shown that nozzles at the conditions of Cases 1 and 2 produce significant instability and mixing enhancement. It appears, therefore, that shock instability contributes to mixing enhancement when the shock is strong but is not the sole reason for the mixing enhancement. An additional likely mechanism is the interaction of the shear layers with the series of compressions and expansions that follow the main shock.

\section{Acknowledgments}

We are grateful for the support by the National University of Singapore, Temasek Labs (Contract No. TL/AE/2004/0001, monitored by Dr. Her Mann Tsai). 


\section{References}

1. Papamoschou, D., “Mixing Enhancement Using Axial Flow,” AIAA Paper 2000-0093, Jan 2000.

2. Murakami, E. and Papamoschou, D., “Experiments on Mixing Enhancement in Dual-Stream Jets,” AIAA Paper 20010668, Jan. 2001.

3. Debiasi, M., Dhanabalan, S. S., Tsai, H. M., and Papamoschou, D., “Mixing Enhancement of High-Bypass Turbofan Exhausts via Contouring of Fan Nozzle”, AIAA Paper 2007-4497, June 2007.

4. Papamoschou, D., Zill, A., “Fundamental Investigation of Supersonic Nozzle Flow Separation,” AIAA Paper 20041111, Jan. 2004.

5. Xiao, Q., Tsai, H.M., and Papamoschou, D., "Numerical Investigation of Supersonic Nozzle Flow Separation," AIAA Journal, Vol. 45, No.3, 2007, pp. 532-541.

6. Plotkin, K.J., “Shock Wave Oscillation Driven by Turbulent Boundary-Layer Fluctuations,” AIAA Journal, Vol. 13, No. 8, 1975, pp. 1036-1040.

7. Poggie, J., and Smits, A.J., “Shock Unsteadiness in a Reattaching Shear Layer,” Journal of Fluid Mechanics, Vol. 429, 2001, pp. 155-185.

8. Poggie, J., and Smits, A.J., "Experimental Evidence for Plotkin Model of Shock Unsteadiness in Separated Flow,” Physics of Fluids, Vol. 17, Issue 1, 2005.

9. Dussauge, J.P., Dupont, P., and Debieve, J.F., "Unsteadiness in Shock Wave Boundary Layer Interaction with Separation,” Aerospace Science and Technology, Vol. 10, Issue 2, 2006, pp. 85-91.

10. Bourgoing, A., Reijasse, Ph., “Experimental Analysis of Unsteady Separated Flows in a Supersonic Planar Nozzle,” Shock Waves, Vol. 14, No. 4, 2005, pp. 251-258.

11. Papamoschou, D., and Johnson, A., “Unsteady Phenomena in Supersonic Nozzle Flow Separation,” AIAA Paper 20063360.

12. Johnson, A., "Plume Instability via Shock Induced Nozzle Flow Separation,” M.S. Thesis, University of California, Irvine, 2008.

13. Xiao, Q., Tsai, H.M., and Papamoschou, D., "Numerical Study of Jet Plume Instability from an Overexpanded Nozzle," AIAA-2007-1319, 2007, Reno, NV.

14. Tam, C.K.W., and Hu, F.Q., "Resonant Instability of Ducted Free Supersonic Mixing Layers Induced by Periodic Mach Waves,” Journal of Fluid Mechanics, Vol. 229, 1991, pp. 65-85.

15. Doty, M.J., and McLaughlin, D.K., "Experiments on Mach-Wave Interactions in a Compressible Shear Layer," AIAA Journal, Vol. 38, No. 10, October 2000, pp. 1871-1878. 


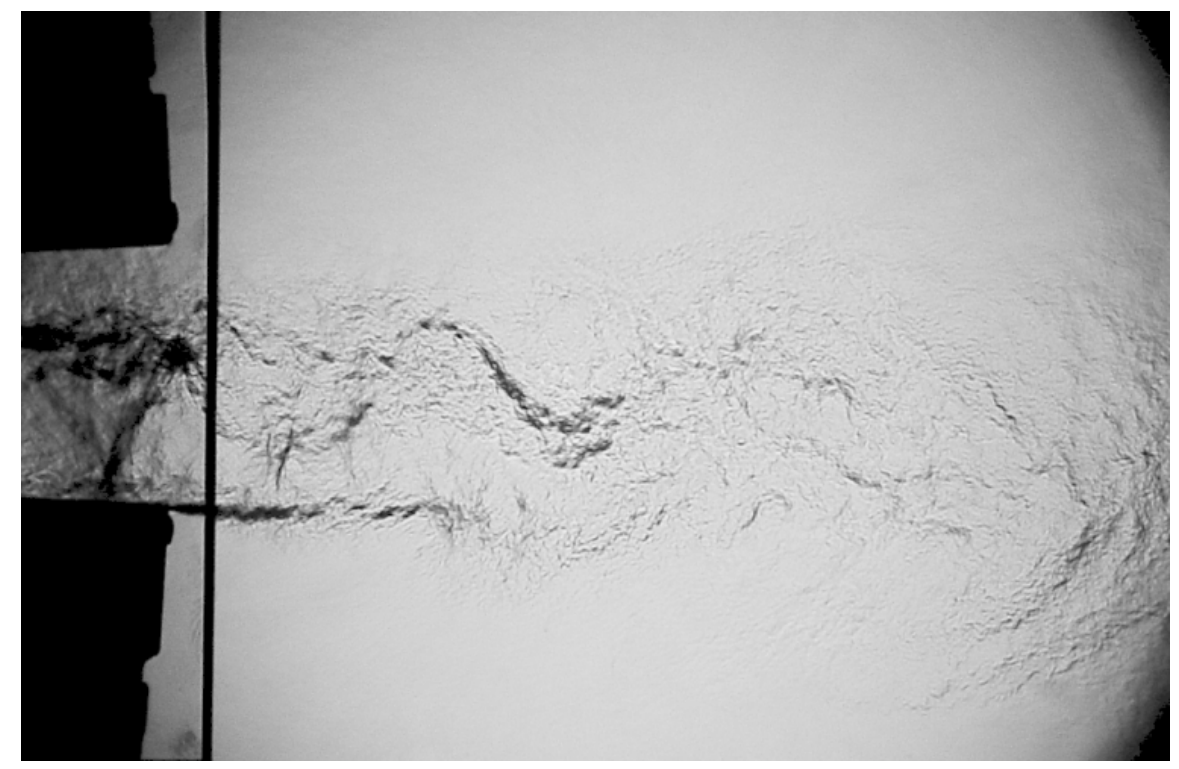

Fig. 1 Plume instability in a nozzle undergoing shock-induced nozzle flow separation.

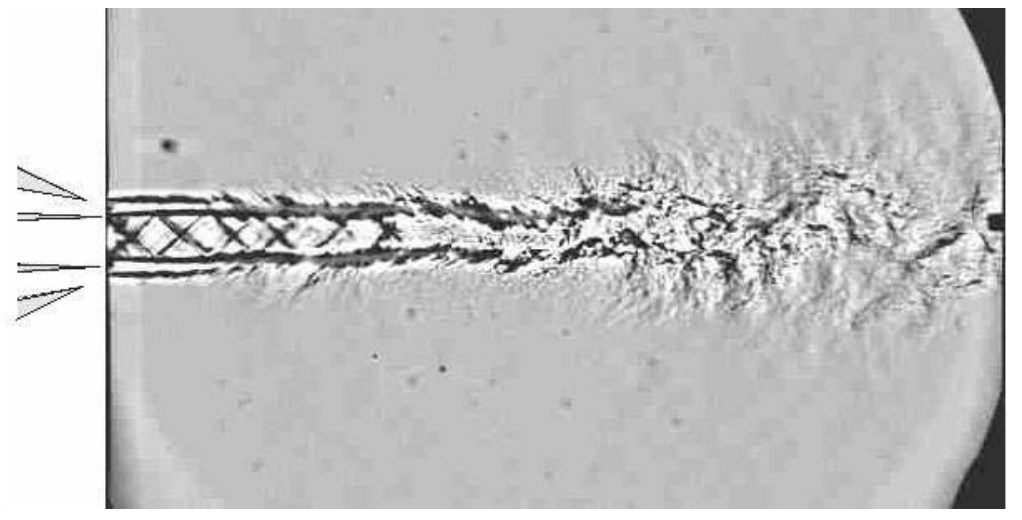

(a)

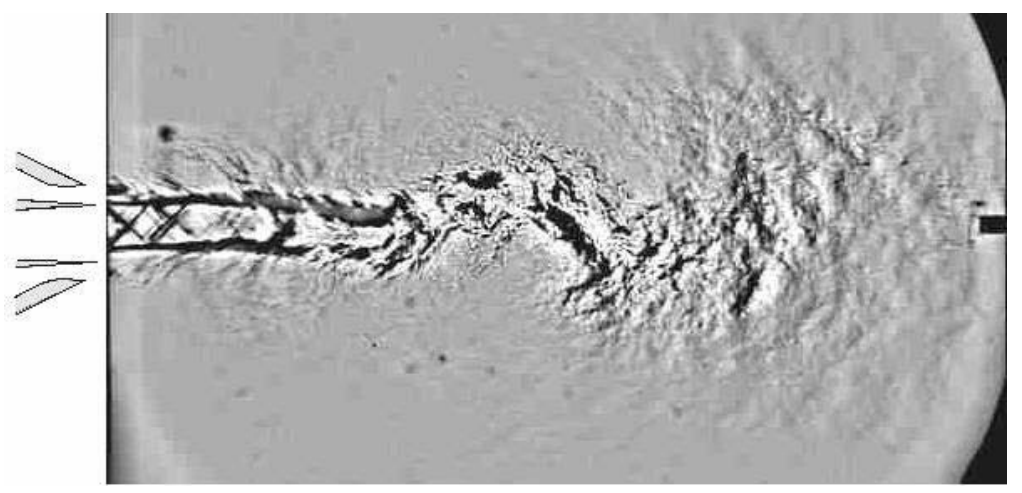

(b)

Fig. 2 Mixing enhancement of a primary jet by changing the contour of the secondary nozzle from convergent (a) to convergent-divergent (b). In the latter case, the secondary nozzle contained a separation shock. 


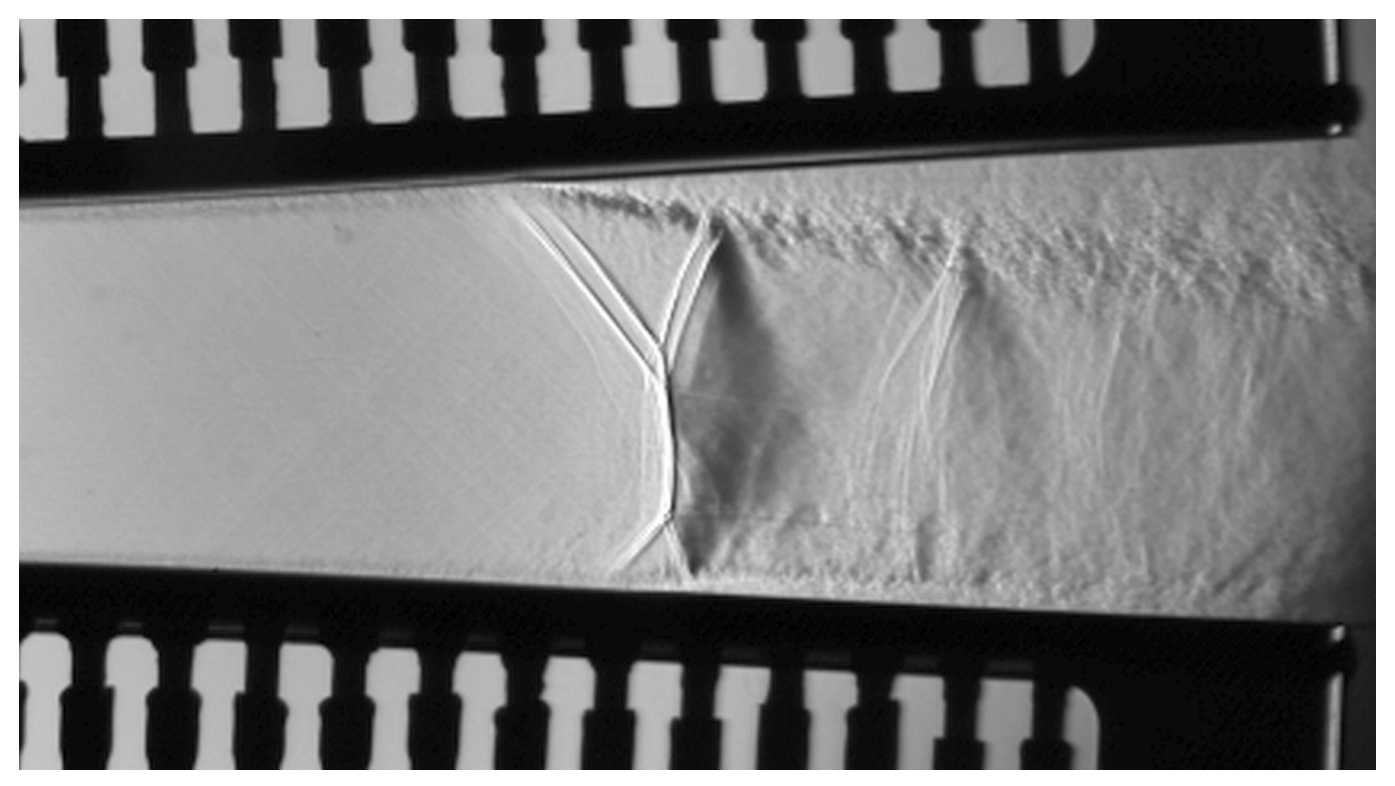

Fig. 3 Schlieren photograph of the internal nozzle flowfield undergoing asymmetric separation.

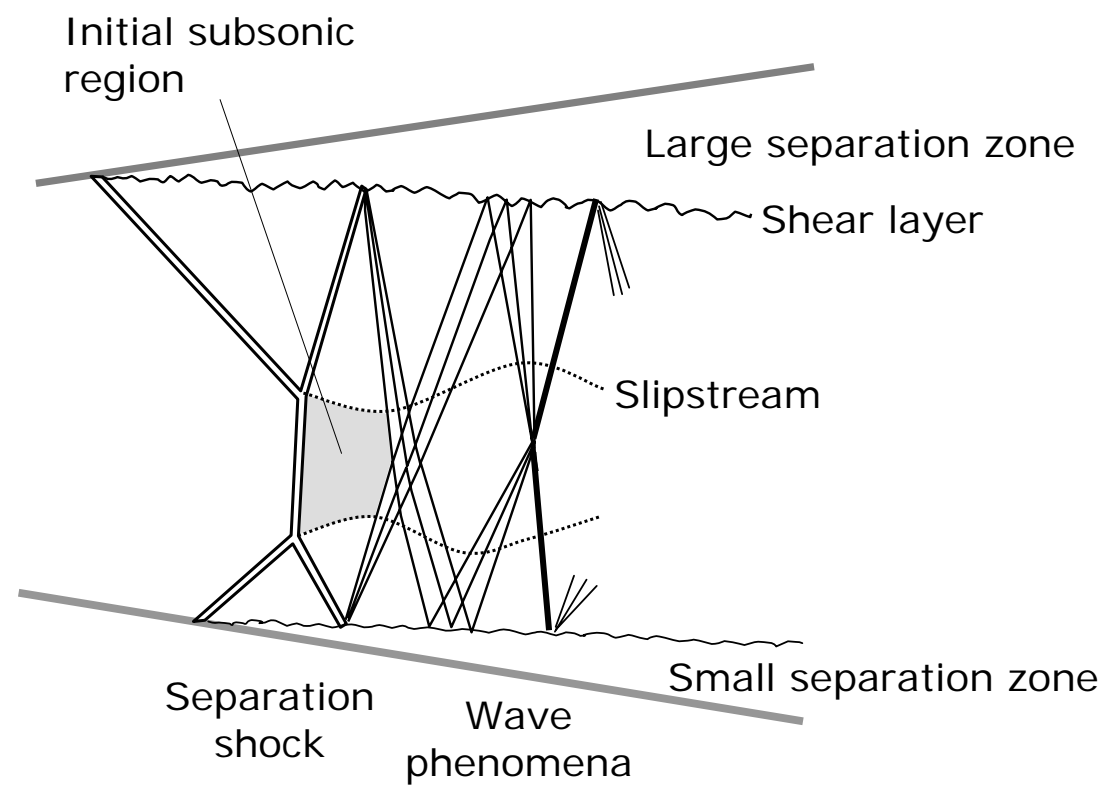

Fig. 4 Schematic of principal phenomena in supersonic nozzle flow separation (based on Ref.4). 


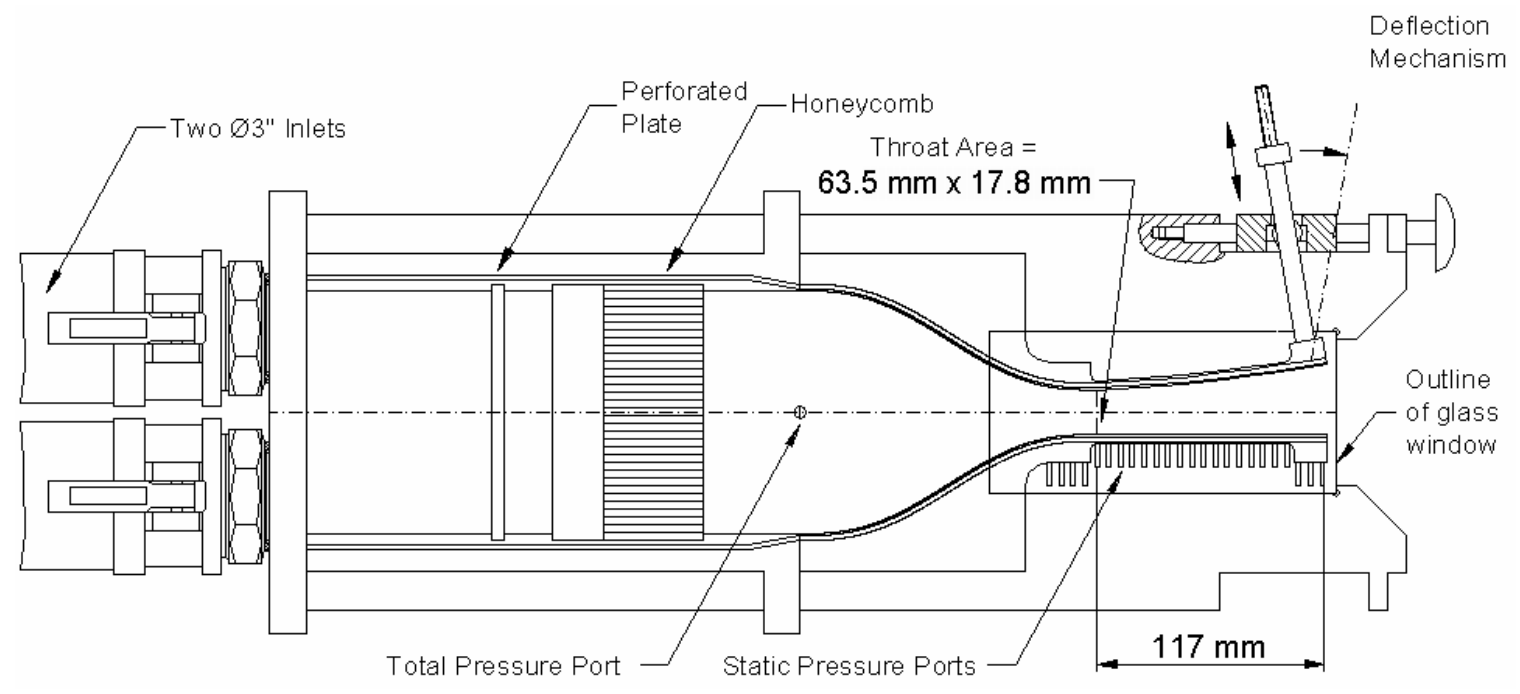

Fig. 5 Schematic of apparatus for the study of supersonic nozzle flow separation and shock unsteadiness.
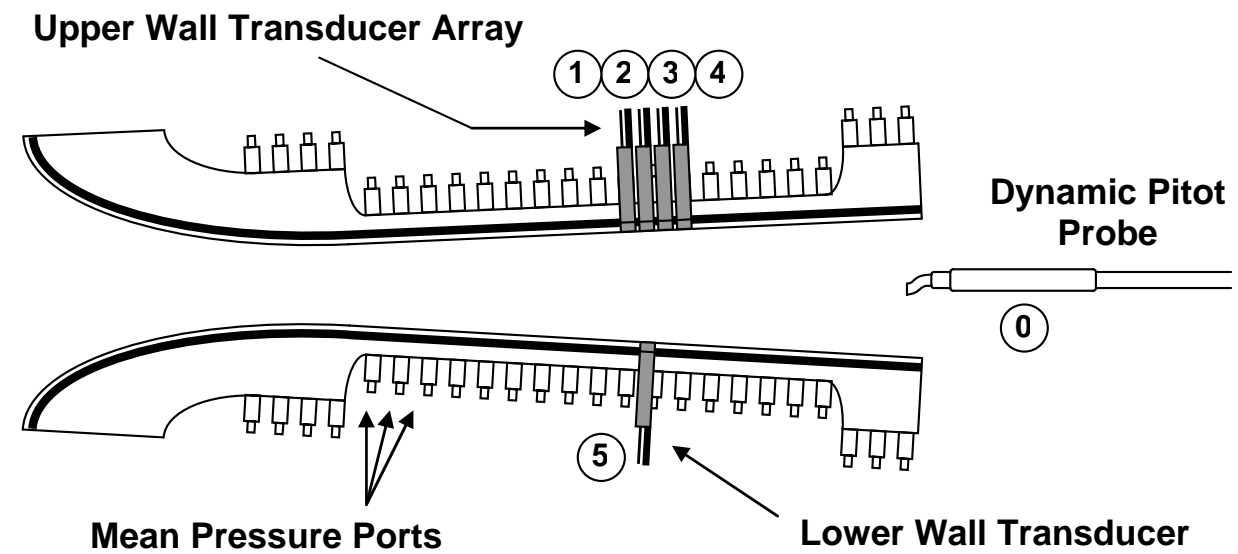

Fig. 6 Diagnostic setup of nozzle.

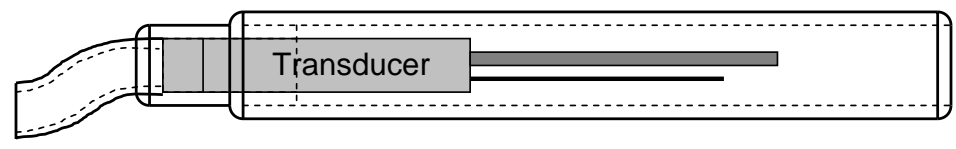

Fig. 7 Design of dynamic Pitot probe, showing protective S-shaped inlet. 

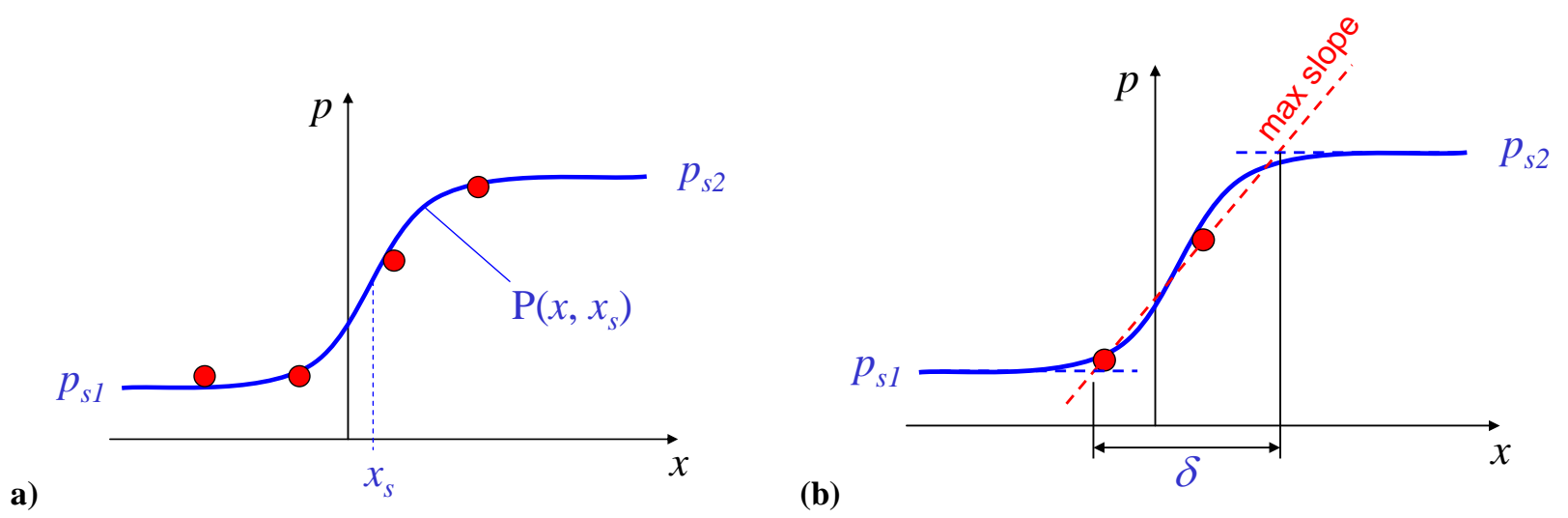

Fig. 8 Illustration of shock fitting routine. (a) Least-squares fit of function $\mathrm{P}\left(x, x_{s}\right)$ through the data; (b) definition of shock thickness.
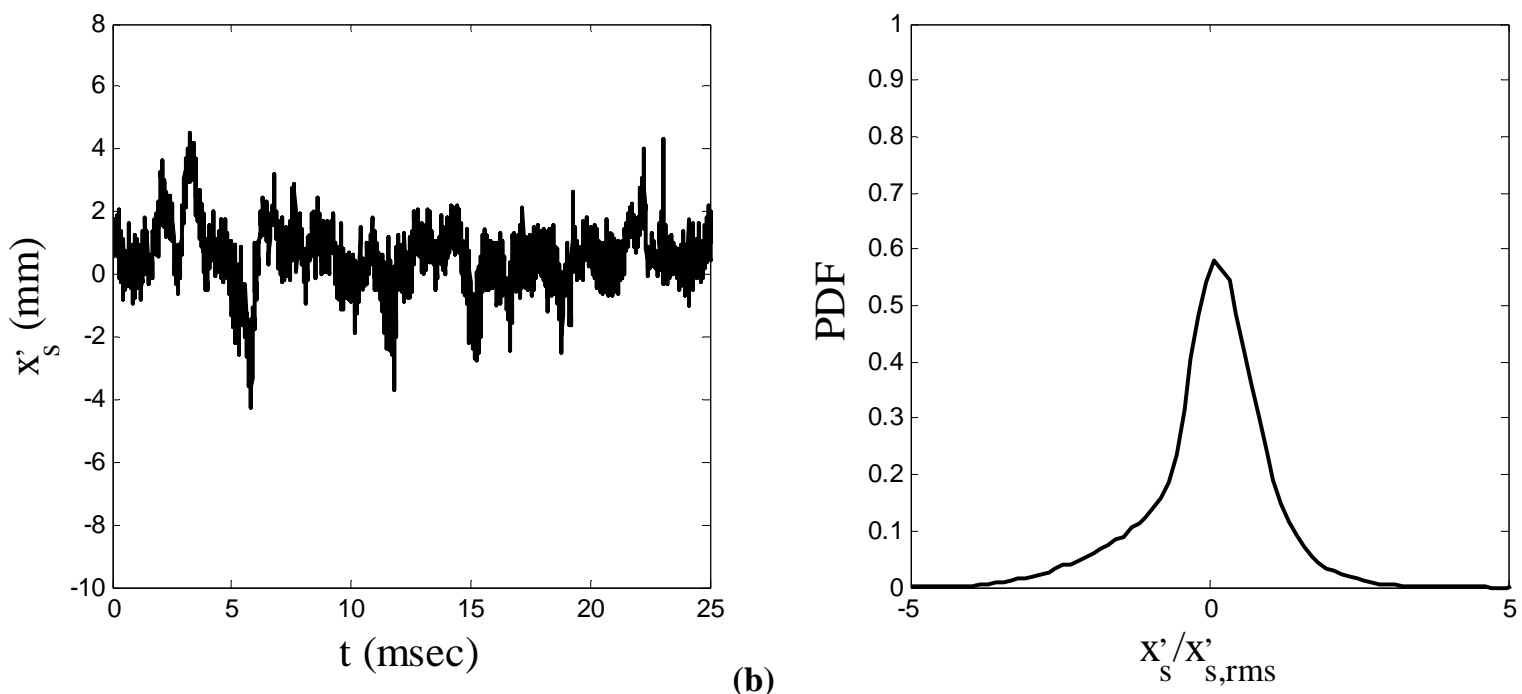

a)

(b)

Fig. 9 Shock-position fluctuation trace (a) and corresponding probability density function (b) for Case 1. 


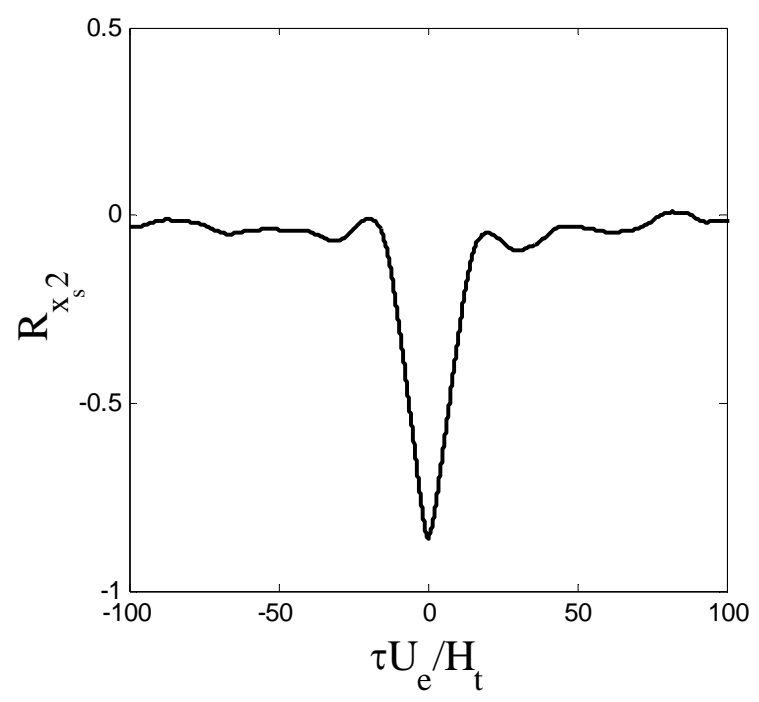

Fig. 10 Normalized cross-correlation between shock position fluctuation and the wall pressure fluctuation at probe 2 for Case 3.

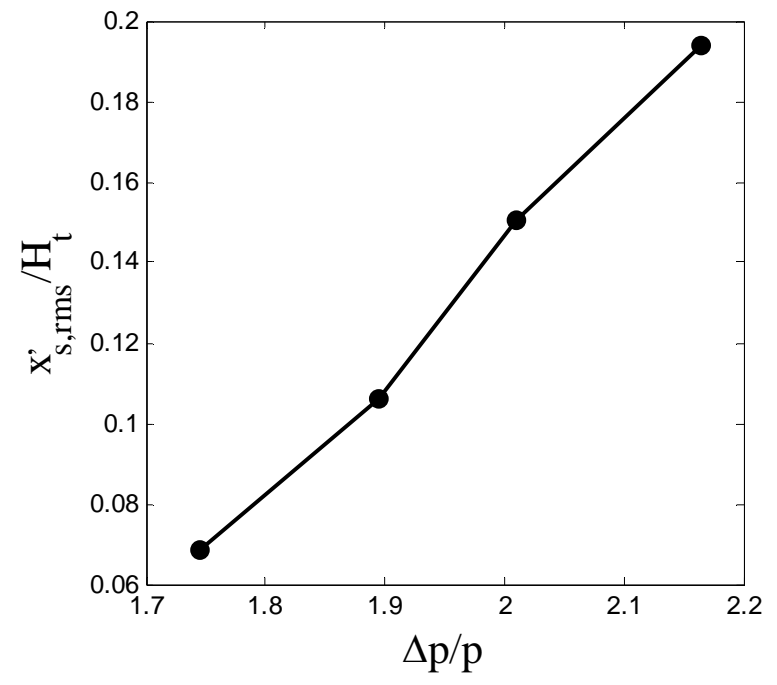

Fig. 12 Root mean square of the shock position fluctuation versus shock strength.

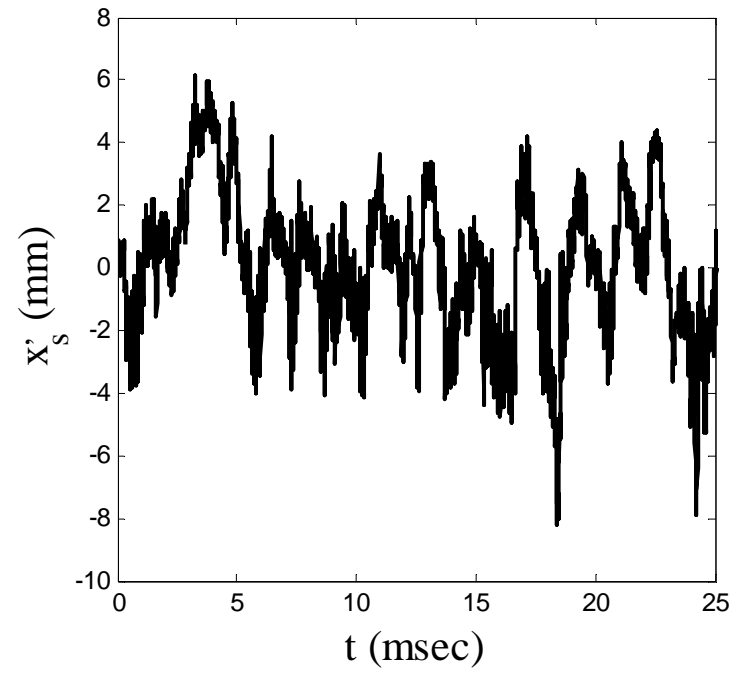

Fig. 11 Shock position fluctuation for case 3 showing increased shock instability.

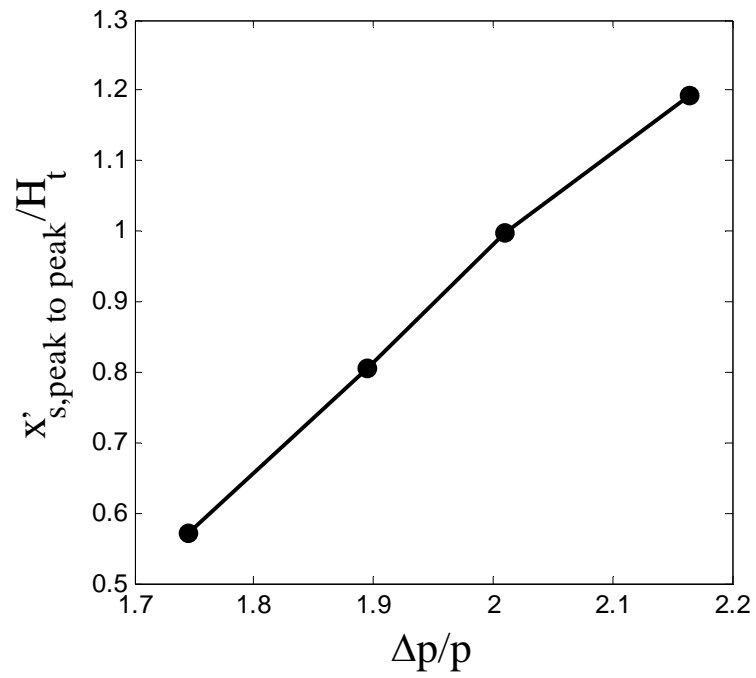

Fig. 13 Peak-to-peak shock displacement versus shock strength. 

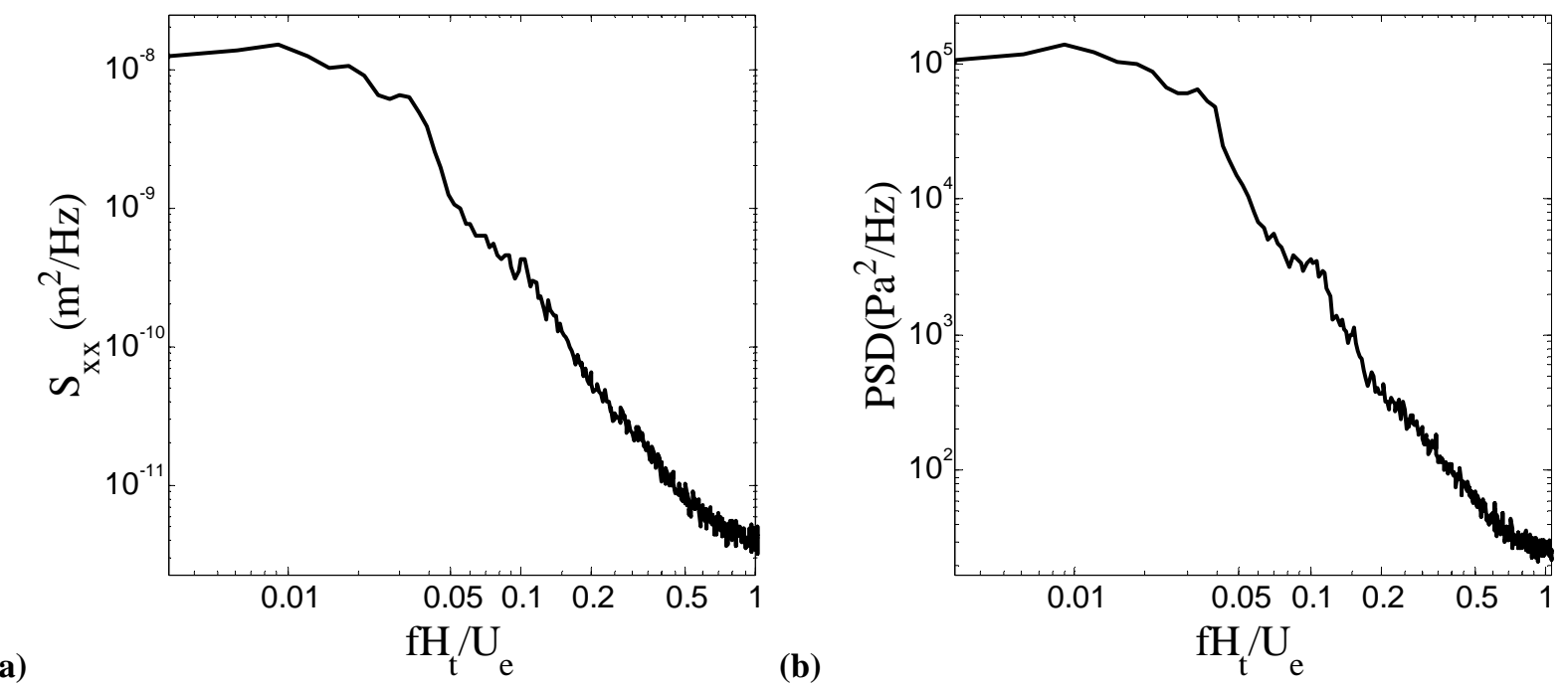

Fig. 14 Autospectra for Case 3: (a) shock position fluctuation; (b) wall pressure fluctuation near the mean shock location.

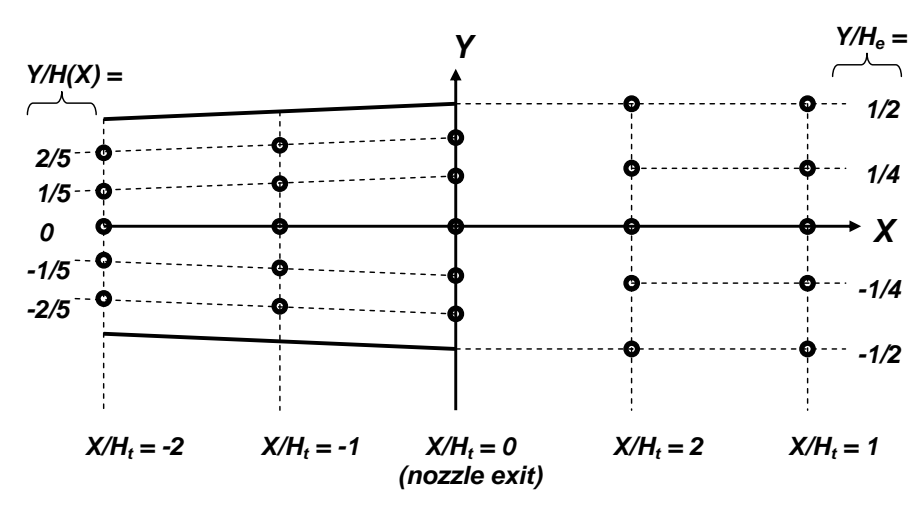

Fig. 15 Coordinate system and positions of Pitot probe.

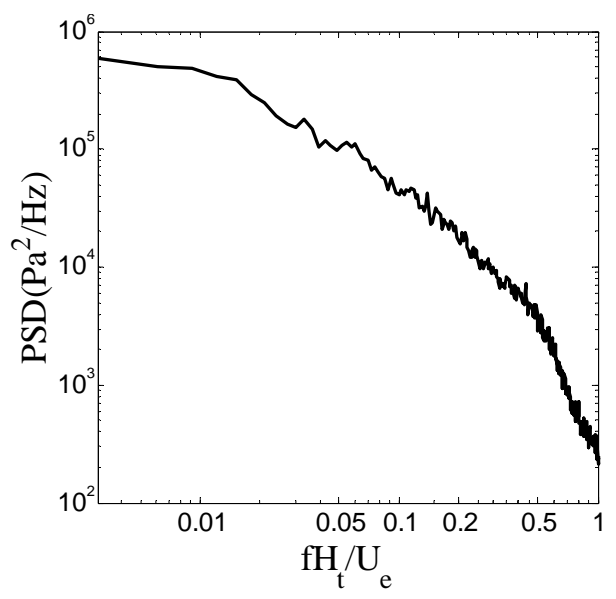

Fig. 16 Autospectrum of total-pressure fluctuation for Case 3 at probe position $X / H_{t}=0.0$ and $Y / H_{t}=0.32$. 

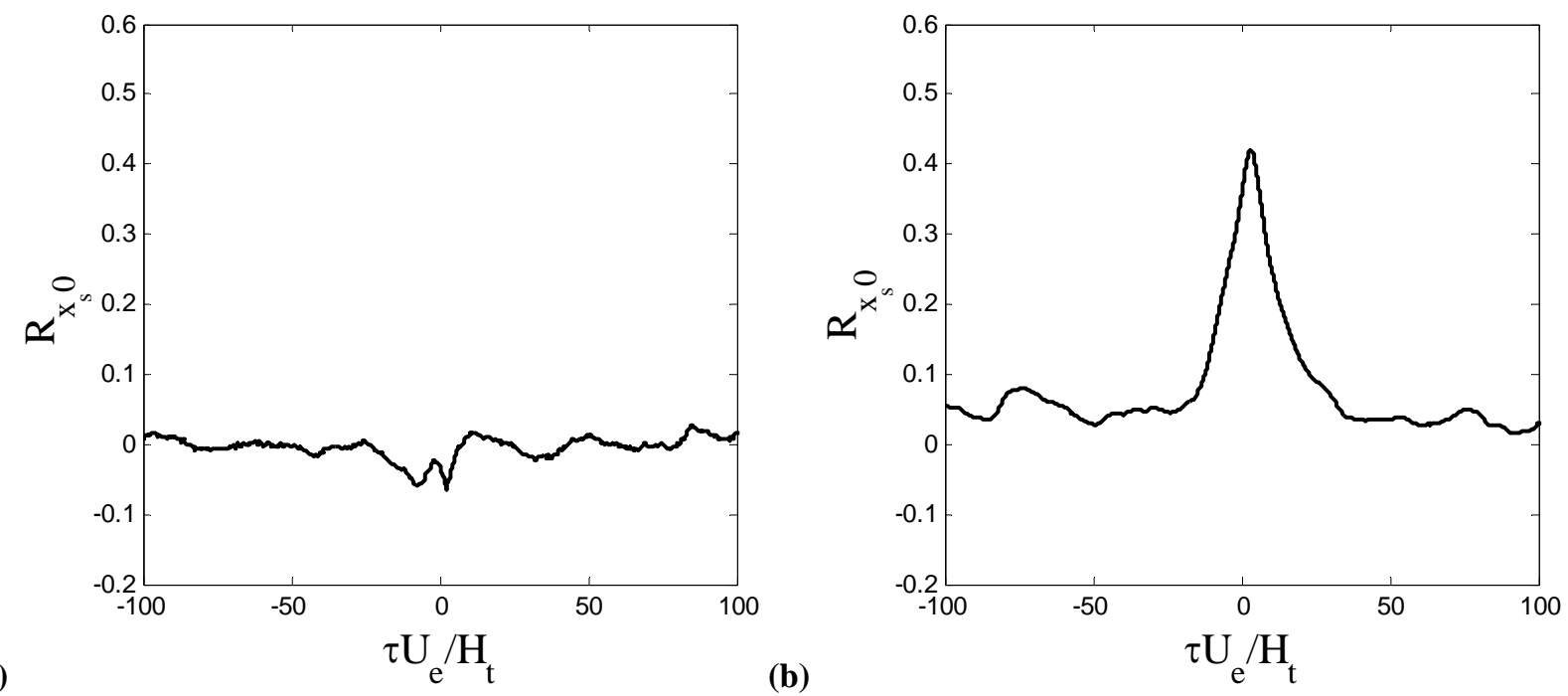

Fig. 17 Cross-correlation of shock position and total-pressure fluctuation measured in the large separation shear layer for: (a) Case 1; (b) Case 3.

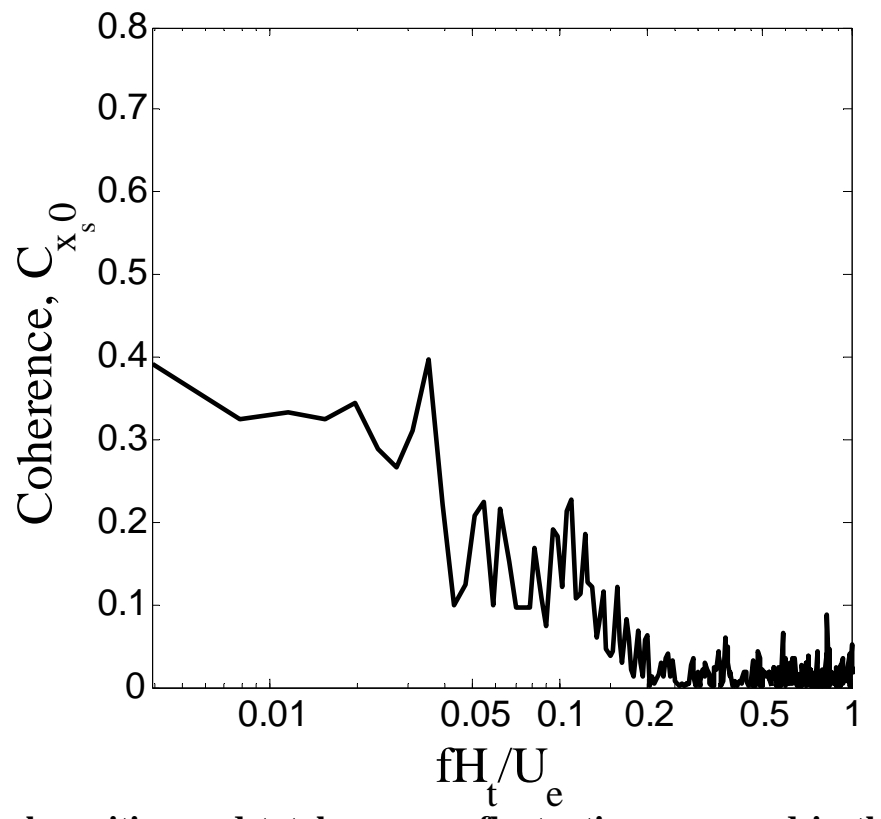

Fig. 18 Coherence of shock position and total-pressure fluctuation measured in the large separation shear layer for Case 3. 


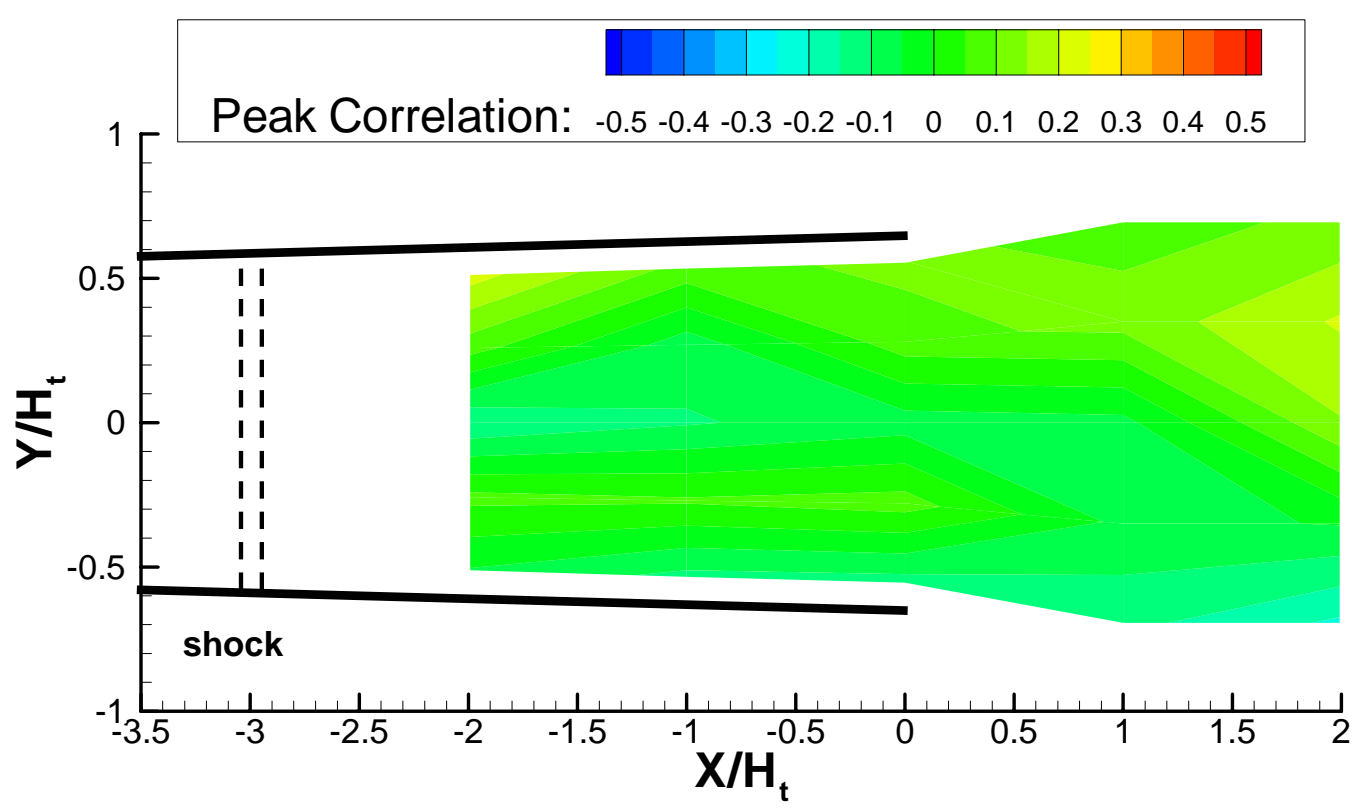

a)

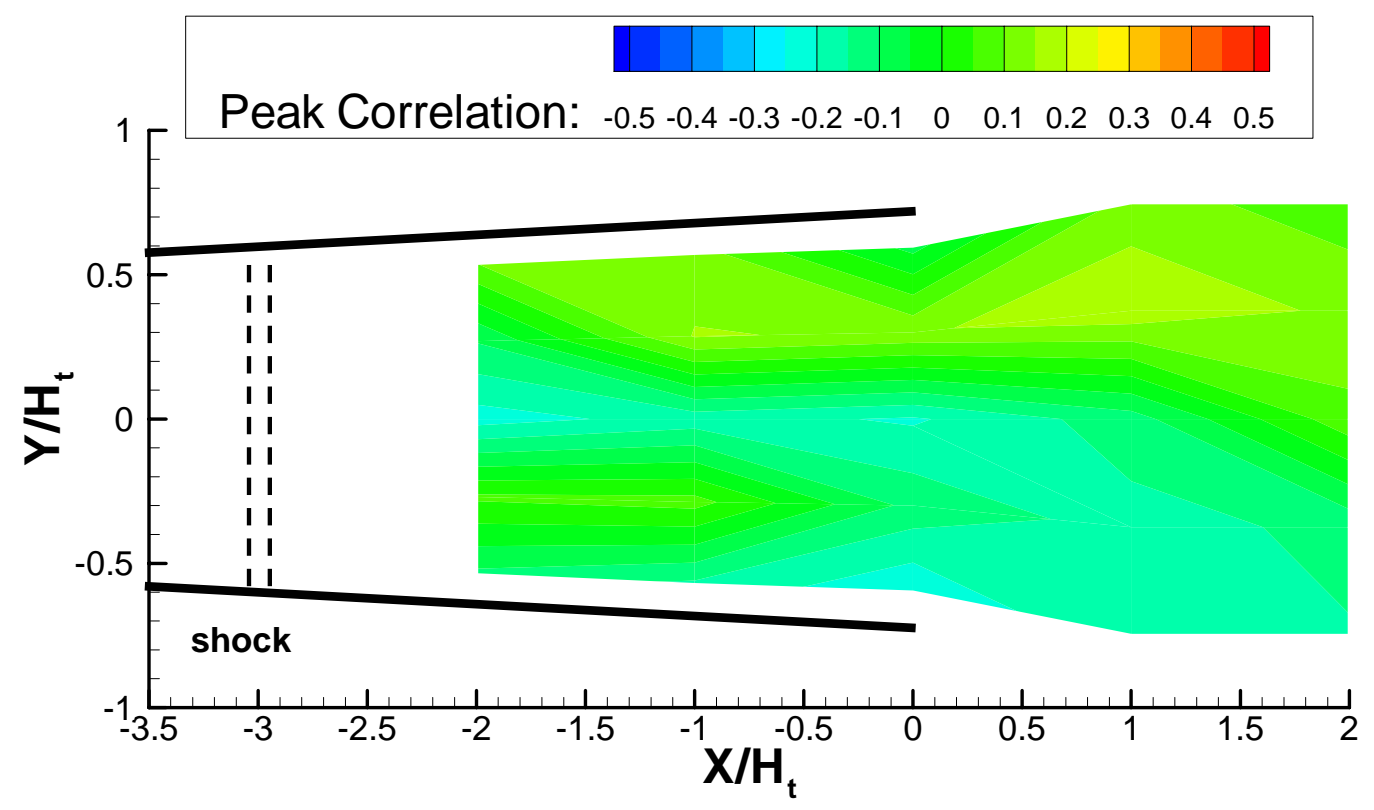

b)

Fig. 19 (continues on next page). 


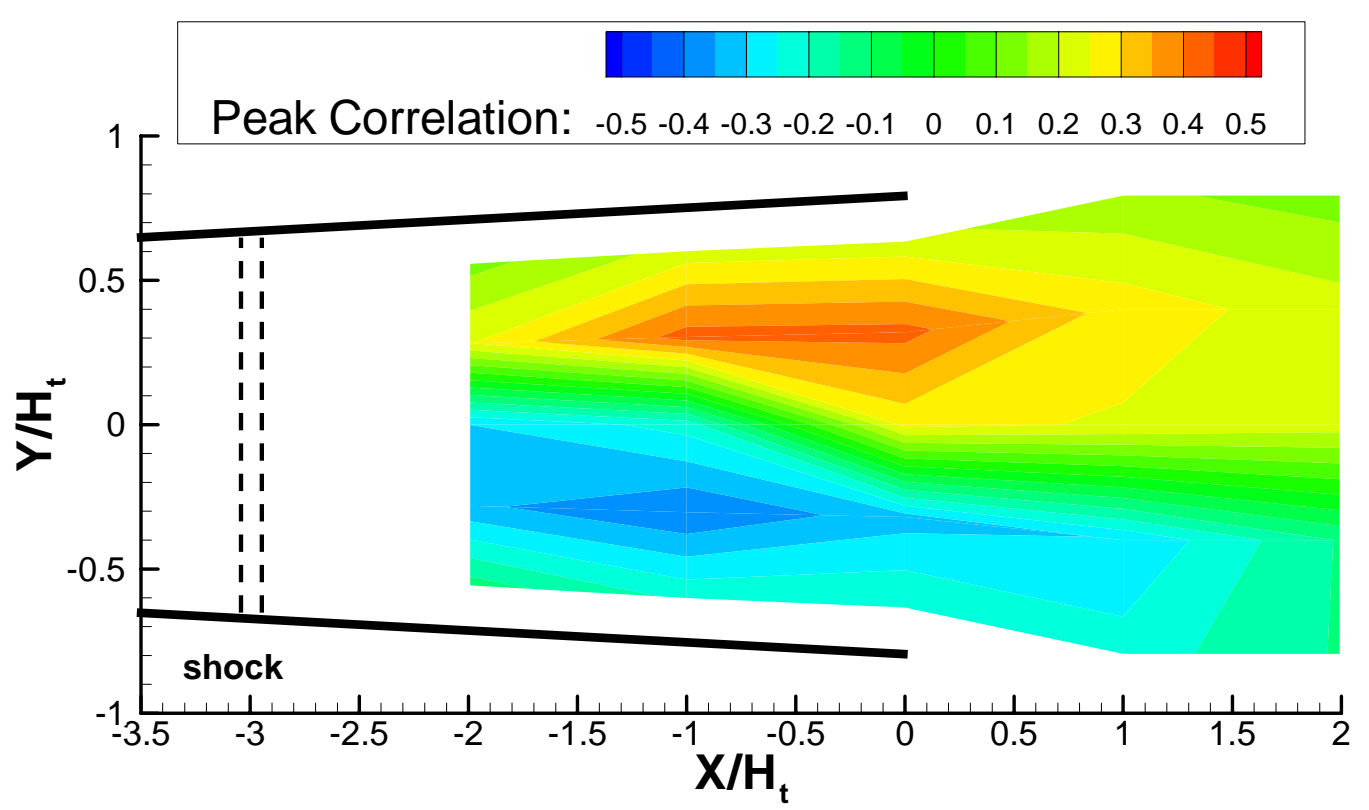

c)

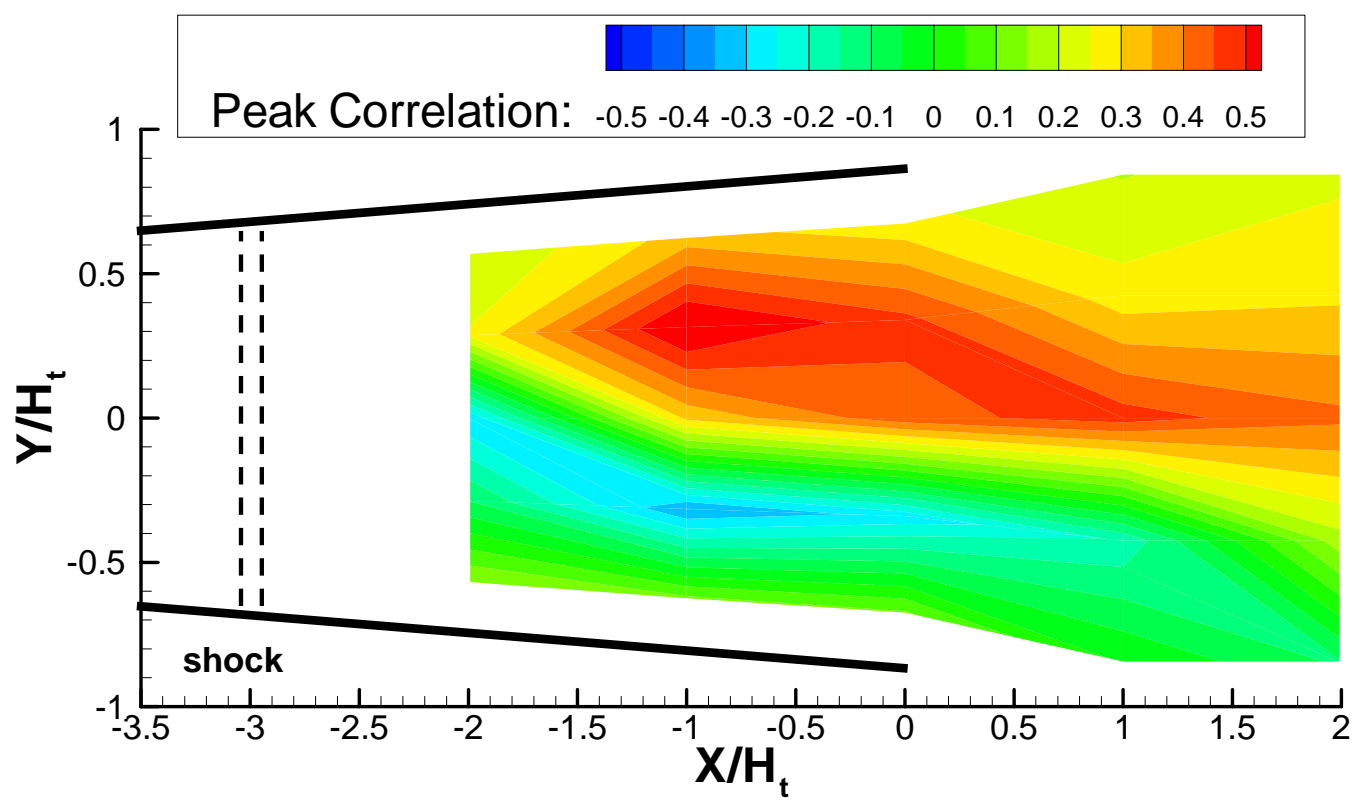

d)

Fig. 19 Contour plots of peak $x_{s}-p_{0}$ ' correlation for: (a) Case 1; (b) Case 2; (c) Case 3; (d) Case 4. 


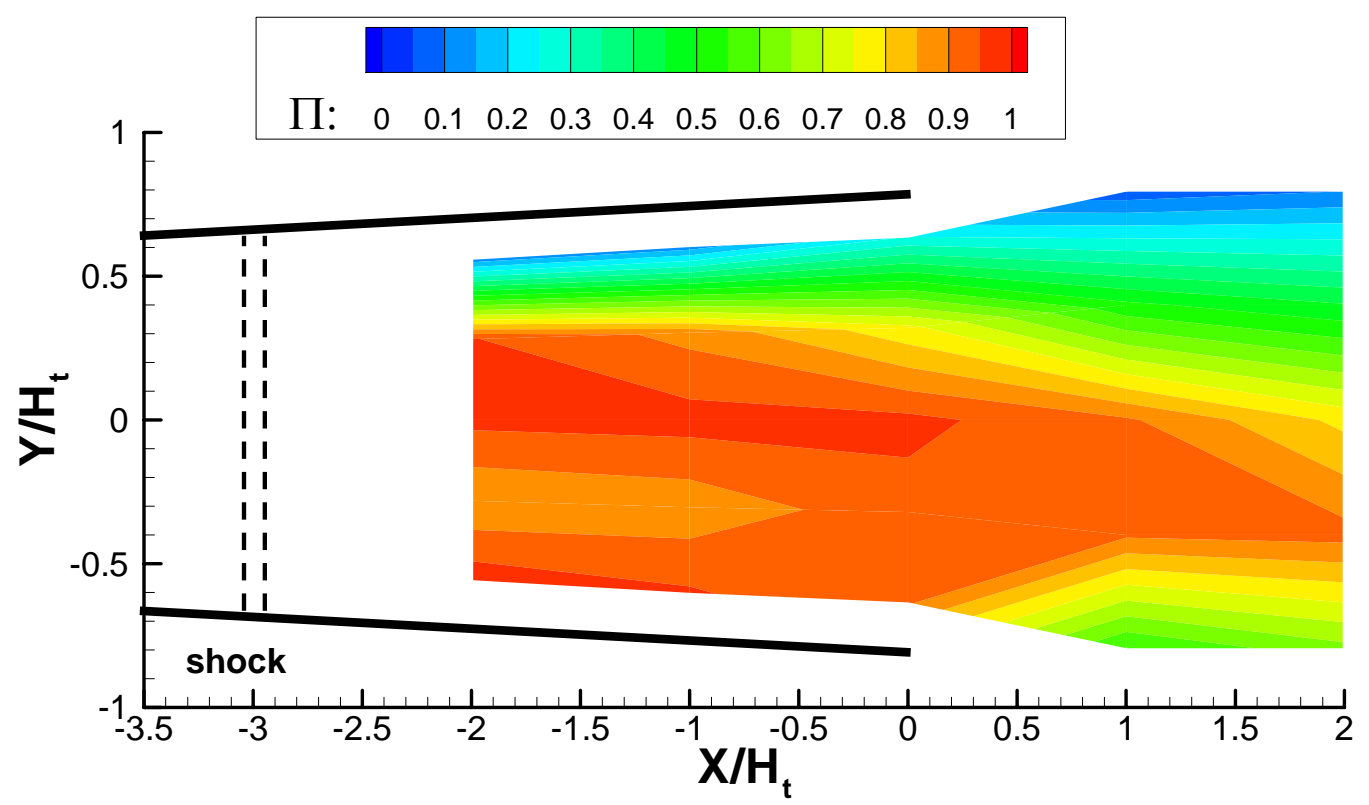

a)

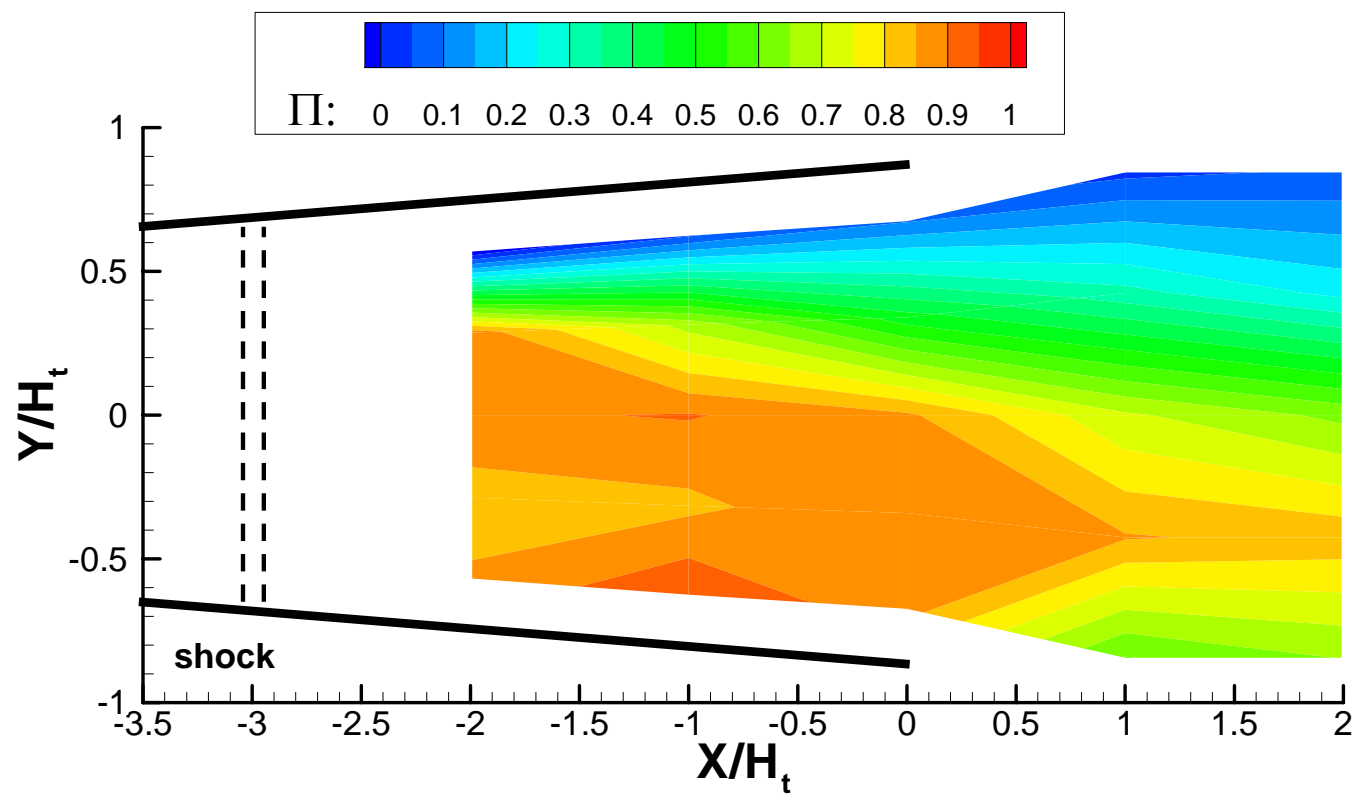

b)

Fig. 20 Contour plots of mean normalized total pressure for: (a) Case 3; (b) Case 4. 